University of Nebraska - Lincoln

DigitalCommons@University of Nebraska - Lincoln

2004

\title{
Two $\Delta$ 9-Stearic Acid Desaturases Are Required for Aspergillus nidulans Growth and Development
}

\author{
Richard A. Wilson \\ University of Nebraska - Lincoln, rwilson10@unl.edu \\ Perng-Kuang Chang \\ USDA-ARS, New Orleans, LA \\ Agnieszka Dobrzyn \\ University of Wisconsin - Madison \\ James B. Ntambi \\ University of Wisconsin - Madison \\ Robert Zarnowski \\ University of Wisconsin - Madison \\ See next page for additional authors
}

Follow this and additional works at: https://digitalcommons.unl.edu/plantpathfungal

Part of the Plant Pathology Commons

Wilson, Richard A.; Chang, Perng-Kuang; Dobrzyn, Agnieszka; Ntambi, James B.; Zarnowski, Robert; and Keller, Nancy P., "Two $\Delta$ 9-Stearic Acid Desaturases Are Required for Aspergillus nidulans Growth and Development" (2004). Fungal Molecular Plant-Microbe Interactions. 11.

https://digitalcommons.unl.edu/plantpathfungal/11

This Article is brought to you for free and open access by the Plant Pathology Department at DigitalCommons@University of Nebraska - Lincoln. It has been accepted for inclusion in Fungal Molecular PlantMicrobe Interactions by an authorized administrator of DigitalCommons@University of Nebraska - Lincoln. 


\section{Authors}

Richard A. Wilson, Perng-Kuang Chang, Agnieszka Dobrzyn, James B. Ntambi, Robert Zarnowski, and Nancy P. Keller 


\title{
Two $\Delta 9$-stearic acid desaturases are required for Aspergillus nidulans growth and development
}

\author{
Richard A. Wilson, ${ }^{\mathrm{a}}$ Perng-Kuang Chang, ${ }^{\mathrm{b}}$ Agnieszka Dobrzyn, ${ }^{\mathrm{c}}$ James M. Ntambi, ${ }^{\mathrm{c}, \mathrm{d}}$ \\ Robert Zarnowski, and Nancy P. Keller ${ }^{\mathrm{a}, *}$ \\ a Department of Plant Pathology, University of Wisconsin-Madison, Madison, WI 53706, USA \\ ${ }^{b}$ Southern Regional Research Center, Agricultural Research Service, US Department of Agriculture, New Orleans, LA 70124, USA \\ ${ }^{\mathrm{c}}$ Department of Biochemistry, University of Wisconsin-Madison, Madison, WI 53706, USA \\ ${ }^{\mathrm{d}}$ Department of Nutritional Sciences, University of Wisconsin-Madison, Madison, WI 53706, USA
}

Received 10 October 2003; accepted 30 December 2003

\begin{abstract}
Unsaturated fatty acids are important constituents of all cell membranes and are required for normal growth. In the filamentous fungus Aspergillus nidulans, unsaturated fatty acids and their derivatives also influence asexual (conidial) and sexual (ascospore) sporulation processes. To investigate the relationship between fatty acid metabolism and fungal development, we disrupted the $A$. nidulans sde $A$ and $s d e B$ genes, both encoding $\Delta 9$-stearic acid desaturases responsible for the conversion of palmitic acid (16:0) and stearic acid (18:0) to palmitoleic acid (16:1) and oleic acid (18:1). The effects of sde $A$ deletion on development were profound, such that growth, conidial and ascospore production were all reduced at 22 and $37^{\circ} \mathrm{C}$. Total fatty acid content was increased over 3-fold in the $\Delta s d e A$ strain, reflected in up-regulation of the expression of the fas $A$ gene encoding the $\alpha$ chain of the fatty acid synthase, compared to wild type. Stearic acid accumulated approximately 3-fold compared to wild type in the $\Delta s d e A$ strain, while unsaturated fatty acid production was decreased. In contrast, disruption of $s d e B$ reduced fungal growth and conidiation at $22^{\circ} \mathrm{C}$, but did not affect these processes at $37^{\circ} \mathrm{C}$ compared to wild type. Interestingly, ascospore production was increased at $37^{\circ} \mathrm{C}$ for $\Delta s d e B$ compared to wild type. Total fatty acid content was not increased in this strain, although stearic acid accumulated 2-fold compared to wild type, and unsaturated fatty acid production was decreased. Combining the $\Delta s d e A$ and $\triangle s d e B$ alleles created a synthetic lethal strain requiring the addition of oleic acid to the medium for a modicum of growth. Taken together, our results suggest a role for $s d e A$ in growth and development at all temperatures, while $s d e B$ is involved in growth and development at lower temperatures.
\end{abstract}

(c) 2004 Elsevier Inc. All rights reserved.

Keywords: Aspergillus nidulans; Fatty acid biosynthesis; $\Delta 9$-Stearic acid desaturase; Fungal development; Stearic acid

\section{Introduction}

Oleic and linoleic acid are the most common unsaturated fatty acid components of Aspergillus nidulans (Calvo et al., 2001; Evans et al., 1986). The previously described $\Delta 12$-oleic acid desaturase (OdeA) of $A$. nidulans converts monounsaturated oleic acid $(18: 1)$ to polyunsaturated linoleic acid (18:2) (Calvo et al., 2001), while the activities of $\Delta 9$-stearic acid desaturase nominally produce oleic acid (18:1) from unsaturated stearic acid (18:0). $\Delta 9$-Stearic acid desaturases additionally

\footnotetext{
${ }^{*}$ Corresponding author. Fax: 1-608-263-2626.

E-mail address:npk@plantpath.wisc.edu (N.P. Keller).
}

function to convert palmitic acid (16:0) to palmitoleic acid (16:1). Studies of many fungal genera clearly show that fatty acid composition is important for normal growth and survival (Chattopadhyay et al., 1985; Scott, 1977; Stukey et al., 1989), with unsaturated fatty acids being critical for maintaining cell membrane fluidity, and hence cell viability, at low temperatures. In the genus Aspergillus, biochemical data suggests an additional requirement of unsaturated fatty acids and their derivatives for the production of multicellular developmental structures such as conidiophores, cleistothecia, and sclerotia (Calvo et al., 1999).

To elucidate the role of unsaturated fatty acids and their derivatives on fungal development, knowledge of 
the genetics and regulation of fatty acid metabolism is required. The importance of polyunsaturated fatty acids for normal Aspergillus fungal development has already been demonstrated by the A nidulans $\Delta$ ode $A$ mutant, where loss of OdeA function leads to accumulation of large amounts of oleic acid and its derivatives, abolishment of polyunsaturated fatty acid production, and a concomitant aberration in both sexual and asexual development (Calvo et al., 2001).

We undertook to create additional fatty acid mutants of $A$. nidulans. Two $\Delta 9$-stearic acid desaturase encoding genes, sde $A$ and $s d e B$, were disrupted. Both mutations decreased unsaturated fatty acid production and increased saturated fatty acid accumulation. $\Delta s d e A$, the most severe mutation, led to up-regulation of a fatty acid synthase gene, fas $A$, and produced 3.5-fold more fatty acids than wild type. $s d e B$ gene expression was also up-regulated in this $\triangle s d e A$ strain. From the results presented below, we see that the sde $A$ and $s d e B$ genes play differing roles in fungal growth and development, with sde $A$ important for fungal viability at all temperatures studied, while $s d e B$ is mainly required for optimal growth and development at lower temperatures.

\section{Materials and methods}

\subsection{Fungal strains and growth conditions}

Aspergillus nidulans strains used in this study are listed in Table 1. Cultures were maintained on glucose minimal medium (GMM) (Calvo et al., 2001) at $37^{\circ} \mathrm{C}$. For asexual conidial production, cultures were grown on $\mathrm{GMM}$ at 22 or $37^{\circ} \mathrm{C}$ in continuous white light using an incubator equipped with a General Electric $15-\mathrm{W}$ broad spectrum fluorescent light bulb (F15T12CW) positioned $50 \mathrm{~cm}$ from the agar surface with a light intensity of $66 \mathrm{mE} / \mathrm{m}^{2} / \mathrm{s}$. For sexual ascospore production, cultures

Table 1

Fungal strains used in this study

\begin{tabular}{|c|c|c|}
\hline Strain & Genotype & Source \\
\hline FGSC773 & wA3; pyrG89; pyroA4; veA1 & FGSC $^{\mathrm{a}}$ \\
\hline TRAW50.410 & $\begin{array}{l}\text { wA3; pyrG89; pyro } A 4 ; \\
\text { AsdeA::pyrG; veA1 }\end{array}$ & This study \\
\hline RRAW6.1 & biA1; pyrG89; $\triangle$ sdeA::pyrG & This study \\
\hline RRAW6.2 & pyrG89; $\triangle$ sdeA::pyrG & This study \\
\hline RDIT9.32 & Prototroph & This study \\
\hline RDIT1.1 & $\arg B 2 ;$ pyrG89; metG81 & D. Tsitsigiannis \\
\hline TRAW128 & $\begin{array}{l}\arg B 2 ; \operatorname{pyr} G 89 ; \triangle s \text { deB::met } G \\
\operatorname{met} G 81\end{array}$ & This study \\
\hline RRAW20 & $\Delta s d e B::$ met $G ;$ met $G 81$ & This study \\
\hline RRAW20.2 & $\begin{array}{l}\triangle s d e A:: p y r G ; \text { pyrG89; } \\
\Delta s d e B:: \text { met } G ; \text { met } G 81\end{array}$ & This study \\
\hline RRAW5.2 & $\triangle \operatorname{ode} A: \because \arg B ; \arg B 2$ & This study \\
\hline
\end{tabular}

${ }^{\mathrm{a}}$ Fungal Genetics Stock Center (Kansas City, KS). were grown in the dark at 22 or $37^{\circ} \mathrm{C}$ on YGT medium (Calvo et al., 2001). These temperatures were chosen because $37^{\circ} \mathrm{C}$ is the optimum growth condition for $A$. nidulans, while $22^{\circ} \mathrm{C}$ represents a significantly lower than optimal temperature suitable for studying the effects of the desaturase mutations on cold tolerance. For RNA studies $10^{6}$ spores of each strain were inoculated into $50 \mathrm{ml}$ flasks of liquid GMM and incubated at $37^{\circ} \mathrm{C}$ with vigorous shaking at $220 \mathrm{rpm}$. Mycelium was harvested at the given time points by vacuum filtration through sterile filter paper.

\subsection{Molecular biology techniques}

For Southern analysis, $10 \mu \mathrm{g}$ of restriction digested genomic DNA was separated by gel electrophoresis in a $1 \%$ agarose gel and transferred by capillary action to Hybond membrane (Amersham).

Total RNA was extracted from mycelia using Trizol reagent (Life Technologies). Ten micrograms of RNA was separated on a $1.2 \%$ agarose- $1.5 \%$ formaldehyde gel. RNA was transferred to Hybond membrane (Amersham) by capillary action. Northern and Southern blot analysis of the $s d e A$ gene was performed using a radiolabelled $1.8 \mathrm{~kb} E c o \mathrm{RI}-X h o \mathrm{I}$ fragment from the $s d e A$ cDNA clone pj5f02a1. Northern and Southern blot analysis of the $s d e B$ gene was performed using a radiolabelled $1 \mathrm{~kb}$ DNA fragment amplified from genomic DNA by the primers 5'sdeBsacIF (see below) and sdeBR1 (GGAGAGGAG CTGGCAAACAG). fas $A$ expression was analyzed using a $0.5 \mathrm{~kb}$ PCR ampified fragment of fas $A$ (GenBank Accession No. U75347) generated from genomic DNA with the primers fasAF (GGATTCCACAGCGG) and fasAR (GGGAGCACG GAGAG). fas $A$ encodes the $\alpha$-subunit of fatty acid synthase and is exclusively involved with the primary metabolism of fatty acids (Brown et al., 1996).

DNA fragments to use as probes were radiolabeled with ${ }^{32} \mathrm{P}$ using the random primer method (Sambrook et al., 1989). Following prehybridization and addition of the probe, the membranes were hybridized overnight at $60^{\circ} \mathrm{C}$, and washed with increasing stringency up to $0.1 \times \mathrm{SSC}, 0.1 \% \mathrm{SDS}$ at $60^{\circ} \mathrm{C}$.

The sde $A$ and $s d e B$ genes were sequenced using previously described techniques and software (Calvo et al., 2001).

PCR conditions were $96^{\circ} \mathrm{C}$ for $3 \mathrm{~min}$, followed by 40 cycles of $96^{\circ} \mathrm{C}$ denaturation $(30 \mathrm{~s}), 55^{\circ} \mathrm{C}$ annealing ( $1 \mathrm{~min})$ and $74^{\circ} \mathrm{C}$ extension $(2 \mathrm{~min})$, unless otherwise stated.

\subsection{Identification of the $\Delta-9$ stearic acid desaturase encoding genes, sde $A$ and sdeB, from $A$. nidulans}

The sde $A$ EST clone pj5f02al (Calvo et al., 2001) was used to probe the pWE15 and pLORIST A. nidulans genomic cosmid libraries (Fungal Genetics Stock Cen- 
ter, Kansas City, KS). Two cosmids, pWBH6 from pWE15 and pLAO12 from pLORIST, were isolated and sequenced using primers designed from the cDNA. The sequence of the sdeA gene has been deposited with GenBank under the Accession No. AF510861. This genomic sequence was then blasted against $A$. nidulans sequences deposited at the Whitehead Institute website (http://www-genome.wi.mit.edu/annotation/fungi/aspergillus/index.html) to identify the coding region of $s d e B$, Accession No. AY504633.

\subsection{Construction of the sde $A$ and $s d e B$ gene disruption vectors}

The sde $A$ disruption vector was designed as follows. First, a $2.2 \mathrm{~kb} E c o$ RI-BamHI fragment from the pPYRG plasmid, containing the $A$. nidulans pyrG gene (Oakley et al., 1987), was ligated into pK19 previously digested with EcoRI and BamHI to give pRAW12. Next, the primer pairs 5'EIF (CCAGCCAGCAGGAATTCAGGAG TAATAAC) and 5'EIR (TGCTATTTCCGCCCGAAT TCTGGAACGTGA) were used to PCR amplify a $1.1 \mathrm{~kb}$ region $5^{\prime}$ to the $s d e A$ gene using Thermolase Taq polymerase (Invitrogen). $5^{\prime} \mathrm{EIF}$ and $5^{\prime} \mathrm{EIR}$ were designed to introduce $E c o$ RI sites at either end of the PCR product, enabling the fragment to be subcloned into pRAW12 at the EcoRI site to give pRAW13. Finally, the primer pairs 3'BIF (GTCTATGGGATCCTGCTCGTACTCCGCC AC) and 3'BIR (CTACACCGACAGGGATCCACAA ACTCCGC) were used to amplify a $1.1 \mathrm{~kb}$ region of the sde $A$ located $700 \mathrm{bp}$ downstream from the translation start site. $3^{\prime} \mathrm{BIF}$ and $3^{\prime} \mathrm{BIR}$ were designed to introduce Bam HI sites at the ends of the PCR fragment, enabling the fragment to be subcloned into pRAW13 at the BamHI site to give pRAW14. pRAW14 represents a disruption vector containing $1.1 \mathrm{~kb}$ each of $5^{\prime}$ upstream and $3^{\prime}$ coding regions of the sde $A$ gene, with $700 \mathrm{bp}$ of coding region, including the translation start site, replaced by the $\operatorname{pyr} G$ cassette. pRAW14 was used to transform the recipient strain FGSC773 using standard techniques (Calvo et al., 2001), and transformants of this auxotrophic strain were initially screened by selection for $p y r G$ prototrophs.

The homology of $s d e B$ to other known $\Delta 9$-stearic acid desaturases allowed us to predict the location of the coding region and design a disruption vector that would insert the met $G$ gene cassette into this region. The sequence of $s d e B$ was used to design PCR primers to construct the $s d e B$ disruption vector as follows: the $5^{\prime}$ end of the $s d e B$ gene was amplified using the primers 5'sdeBsacIF (GGCAGAGCTCGGCGGTTCAGAGA GGCTACCG) and 5'sdeBsacIR (GGCAGAGCTCCC AAGTCCACAGACCAATGTCGG) to give a $1 \mathrm{~kb}$ fragment with $S a c$ I restriction sites introduced at the ends. The plasmid pUG11-41, containing the met $G$ gene including its promoter and terminator regions (Sienko and Paszewski, 1999), and the $s d e B$ PCR fragment were digested with $S a c$ I and the $s d e B 5^{\prime}$ flank was ligated into the pUG11-41 to give pRAW17. Next, the $3^{\prime}$ end of the $s d e B$ gene was amplified by $3^{\prime}$ sdeBsphI (GGACTGCA TGCGGGGCGATTGGCGCGGCGGG) and 3'sdeBHindIII (CGAACCAAGCTTGCCGGGAGAATAGC CTTGCCGGGCC) to give a $1 \mathrm{~kb}$ fragment. pRAW17 and this fragment were digested with $S p h \mathrm{I}$ and HindIII and the $3^{\prime}$ fragment ligated into pRAW17 to give pRAW18. Therefore, pRAW18 represents the coding region of $s d e B$ split in the center by the met $G$ coding region. This vector was used to transform the $\operatorname{met} G$ requiring strain RDIT1.1, and putative disruptants were selected initially by met $G$ prototrophy.

\subsection{Confirmation of sde $A$ and sdeB gene disruption}

Prototrophic transformants were screened for homologous gene replacement of sdeA with $p y r G$ using PCR. PCR conditions were $96^{\circ} \mathrm{C}$ for $3 \mathrm{~min}$, followed by 40 cycles of $96^{\circ} \mathrm{C}$ denaturation $(30 \mathrm{~s}), 52^{\circ} \mathrm{C}$ annealing ( $1 \mathrm{~min}$ ), and $74^{\circ} \mathrm{C}$ extension (5 min) using PCR primers $5^{\prime} \mathrm{EIF}$ and $3^{\prime} \mathrm{BIR}$. Wild type $s d e A$ yielded a $3.1 \mathrm{~kb}$ PCR fragment, while $\Delta s d e A$ yielded a $4.4 \mathrm{~kb}$ fragment. Single copy integration was confirmed by Southern hybridization.

For $s d e B$, disruption of this gene by met $G$ was confirmed using a PCR approach followed by Southern analysis. PCR conditions were $96^{\circ} \mathrm{C}$ for $3 \mathrm{~min}$, followed by 40 cycles of $96^{\circ} \mathrm{C}$ denaturation $(30 \mathrm{~s}), 62^{\circ} \mathrm{C}$ annealing $(1 \mathrm{~min})$, and $74^{\circ} \mathrm{C}$ extension $(4 \mathrm{~min})$ using the primers $5^{\prime}$ sdeBsacIF and sdeBR1. Wild type $s d e B$ gene yielded a $1.5 \mathrm{~kb}$ PCR fragment, the distance between 5 'sdeBsacIF and sdeBR 1 . $\Delta s d e B$ yielded a $4 \mathrm{~kb}$ fragment representing the distance between $5^{\prime}$ sdeBsacIF and sdeBR 1 in addition to the $2.5 \mathrm{~kb}$ met $G$ gene cassette located between these two primers. Single copy integration was confirmed by Southern hybridization.

To ensure the severe phenotype of the $\Delta s d e A$ strain was due solely to the single replacement of this gene by $\operatorname{pyr} G$, the full-length $s d e A$ gene was re-introduced into a $\triangle s d e A$ strain using the vector pRAW19. pRAW19 was constructed as follows: a $2.7 \mathrm{~kb}$ fragment, containing the sde $A$ coding region and upstream elements, was amplified from genomic DNA using $p f u$ proof reading Taq and the primers $5^{\prime} \mathrm{UapCsphIF}$ (CCCGCGGCATGCGA TCAATTTAAGATAAGC) and 3'sdeAR (CCAGCAT GCGGAAGTGGATGGCATAT) to introduce $S p h$ I sites at either end. The resulting PCR fragment and pUG11-41 were digested with $S p h \mathrm{I}$, and the sdeA containing fragment was ligated into this vector to give pRAW19. pRAW19 was then transformed into 4 sde $A$ strain RRAW6.1 and restored $s d e A$ function was selected for by remediated growth and sporulation at lower temperatures $\left(22^{\circ} \mathrm{C}\right)$ compared to wild type, followed by Southern confirmation. Five transformants were single spore purified and analyzed. 
The double mutant $\Delta s d e A ; \Delta s d e B$ was constructed by sexual cross of RRAW6.2 and TRAW128 (Table 1) using standard techniques (Pontecorvo et al., 1953). As mutations in both $\Delta 9$-stearic acid desaturases were lethal, $\Delta s d e A ; \Delta s d e B$ double mutant strains were selected on GMM supplemented with $1 \%(\mathrm{v} / \mathrm{v})$ Tween 80 , and confirmed by PCR.

\subsection{Physiological studies}

Conidiation studies were performed on plates containing $25 \mathrm{ml}$ GMM. For each plate, a $5 \mathrm{ml}$ top layer of cool but molten agar that contained $10^{6}$ spores of the appropriate strain was added. For each strain, there was a minimum of four replicate plates. A core of $15 \mathrm{~mm}$ was removed from the plates at the appropriate time interval, and homogenized in $2 \mathrm{ml}$ of $0.01 \%$ Tween 80 water to release the spores. Colony radial growth was recorded as colony diameter and the experiments were performed in triplicate. To study the remediation of the $\Delta s d e A$ mutation by exogenous oleic acid, $1 \%(\mathrm{v} / \mathrm{v})$ of Tween $80(\mathrm{ICN})$ was added to GMM.

Aspergillus nidulans ascospore production studies were performed on plates containing $30 \mathrm{ml}$ YGT medium (Calvo et al., 2001). To each plate was added a $5 \mathrm{ml}$ top layer of molten agar containing $10^{6}$ spores of the appropriate strain. For each strain, there was a minimum of four replicate plates. Strains were grown in the dark for $240 \mathrm{~h}$ at 37 and $22^{\circ} \mathrm{C}$. A core of $15 \mathrm{~mm}$ was removed from each plate, and homogenized in $2 \mathrm{ml}$ of sterile distilled water to break the cleistothecium and release the spores. Conidia and ascospores were counted on a haemocytometer.

\subsection{Fatty acid analysis}

Fatty acid methyl esters were generated from $A s$ pergillus mycelia for gas chromatography (GC) analysis as follows. After growth in GMM for $72 \mathrm{~h}$ at $37^{\circ} \mathrm{C}$, constant light conditions, mycelia was lyophilized, ground into fine powder, and the lipids extracted three times in chloroform:methanol (2:1) following Bligh and Dyer (1959). About $0.64 \mathrm{ng}$ heptadecanoic acid (C17:0) was added as an internal standard. The samples were dried down, resuspended in $1 \mathrm{ml}$ of $5 \% \mathrm{HCl}$ in $90 \%$ methanol, and placed at $95{ }^{\circ} \mathrm{C}$ for $30 \mathrm{~min}$ to generate fatty acid methyl esters (FAME). The methylating reaction was quenched with water, and the FAMEs extracted three times in hexane. The samples were concentrated, and $1 \mu \mathrm{l}$ of the hexane layer was examined by gas chromatography. Identification of peaks was achieved by comparison of sample retention times to those of palmitic-, palmitoleic-, stearic-, oleic-, linoleic-, and linolenic acid standards (FAME-GC mix, Sigma).

\subsection{Statistical analysis}

Spore data and colony diameters were evaluated by analysis of variance (ANOVA) using SAS.

\section{Results}

3.1. The sdeA and sdeB genes of A. nidulans encode 49stearic acid desaturases

Sequencing of the $A$. nidulans genomic cosmids pWBH6 and pLAO12 by sdeA cDNA specific primers revealed a coding sequence of $1419 \mathrm{bp}$, interrupted by one intron, encoding a 455 amino acid $\Delta 9$-stearic acid desaturase (GenBank sequence Accession No. AF510861). The deduced amino acid sequence has highest identity to the fungal $\Delta 9$-stearic acid desaturase from Ajellomyces capsulata (83\%) (GenBank sequence Accession No. S52745). SdeA contains the conserved histidine clusters, composing the Fe-binding active centers of the enzyme, present in all $\Delta 9$-desaturases (Los and Murata, 1998).

Examination of the Whitehead A. nidulans database with the sdeA genomic sequence revealed a second putative $\Delta 9$-desaturase, sdeB, located on scaffold IV (GenBank sequence Accession No. AY504633) encoding an approximately 460 amino acid protein. It had $55 \%$ identity to sdeA at the nucleotide level and $66 \%$ identity at the amino acid level including the conserved His cluster motifs. In GenBank BLAST analysis, SdeB also had high identity ( $75 \%$ at the amino acid level) to the $\Delta 9$-stearic acid desaturase from Neurospora crassa (GenBank sequence Accession No. XM_324615). Examination of the Whitehead database with either sdeA, $s d e B$ or other known $\Delta 9$-stearic acid desaturases did not produce significant matches to any other gene, indicating that $s d e A$ and $s d e B$ are likely the only $\Delta 9$-stearic acid desaturase encoding genes present in A. nidulans.

The two desaturase genes were disrupted by homologous recombination as described in materials and methods.

For sake of clarity, $A$. nidulans strains carrying the gene mutations $\triangle s d e A$ and $\triangle s d e B$ are referred by these genotypes and not by strain name (i.e., TRAW50.410) for the rest of this presentation.

\subsection{Effect of sdeA and sdeB mutations on A. nidulans conidiospore production at 37 and $22^{\circ} \mathrm{C}$}

In order to compare to previous studies, conidial production was assessed on glucose minimal medium (GMM). Conidia were counted at $72 \mathrm{~h}$. At $37^{\circ} \mathrm{C}, \Delta s d e A$ produced significantly less $(p \leqslant 0.05)$ conidia than wild type and $\Delta s d e B$ strains (Fig. 1). Conidiation of $\Delta s d e B$ 


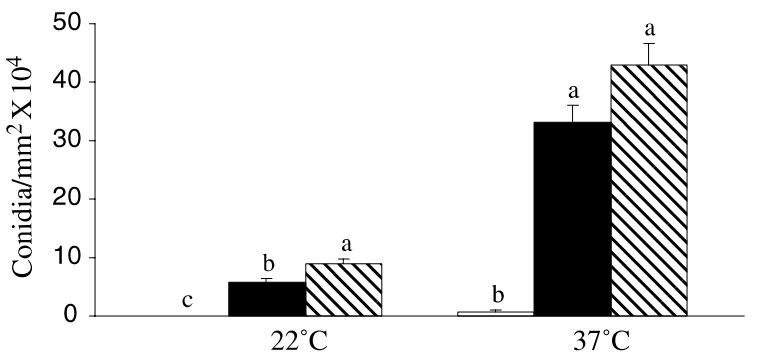

Fig. 1. Production of conidia. Cultures of $\Delta s d e A$ (open column), $\Delta s d e B$ (closed column), and wild type (hatched column) were grown in the light on GMM agar plates at 22 and $37^{\circ} \mathrm{C}$ for $72 \mathrm{~h}$ incubation times. Values of conidia counts are the means of four replicates. Columns with the same letter are not significantly different $(p \leqslant 0.05)$. Conidia counts at 22 and $37^{\circ} \mathrm{C}$ were not compared.

was not significantly different from wild type at $37^{\circ} \mathrm{C}$, however $\triangle s d e B$ conidiated significantly less $(p \leqslant 0.05)$ than wild type when grown at $22^{\circ} \mathrm{C}$ (Fig. 1). $\Delta s d e A$ did not conidiate at this temperature. This reduction in asexual sporulation at $22^{\circ} \mathrm{C}$ of the two mutant strains compared to wild type suggests the importance of unsaturated fatty acids, or at least normal fatty acid profiles, for growth of the fungus at lower temperatures $(p \leqslant 0.05)$.

Conidiation of $\triangle s d e A$ was remediated by transformation with the full-length $s d e A$ gene. This strain neither grew nor developed significantly different $(p \leqslant 0.05)$ to wild type even at $22{ }^{\circ} \mathrm{C}$ (data not shown).

\subsection{Effect of sdeA and sdeB mutations on A. nidulans ascospore production at 37 and $22^{\circ} \mathrm{C}$}

Ascospore production was assessed on YGT medium after $240 \mathrm{~h}$. At $37^{\circ} \mathrm{C}$, ascospore production was significantly $(p \leqslant 0.05)$ reduced in $\Delta s d e A$ compared to $\Delta s d e B$ and wild type. Interestingly, $\triangle s d e B$ ascospore production was significantly increased $(p \leqslant 0.05)$ compared to wild type at this temperature (Fig. 2).

Ascospore production was significantly reduced $(p \leqslant 0.05)$ in $\triangle s d e A$ compared to $\triangle s d e B$ and wild type at

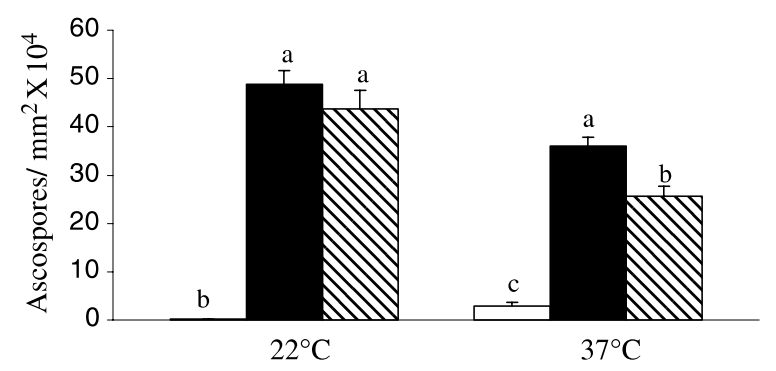

Fig. 2. Production of ascospores. Cultures of A. nidulans 4 sdeA (open column), $\triangle s d e B$ (closed column), and wild type (hatched column) were grown in the dark on YGT agar plates at 22 and $37^{\circ} \mathrm{C}$ for $240 \mathrm{~h}$ incubation times. Values of ascospore counts are the means of four replicates. Columns with the same letter are not significantly different $(p \leqslant 0.05)$. Ascospore counts at 22 and $37^{\circ} \mathrm{C}$ were not compared.

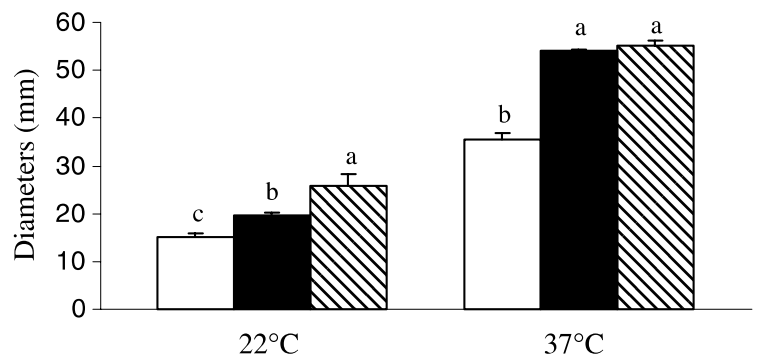

Fig. 3. Radial colony growth. Cultures of $A$. nidulans $\triangle s d e A$ (open column), $\Delta s d e B$ (closed column), and wild type (hatched column) were grown on GMM agar plates at 22 and $37^{\circ} \mathrm{C}$ for $120 \mathrm{~h}$ in the light. Values for radial colony diameters are the means of three replicates. Columns with the same letter are not significantly different $(p \leqslant 0.05)$. Radial colony diameters at 22 and $37^{\circ} \mathrm{C}$ were not compared.

$22^{\circ} \mathrm{C}$ (Fig. 2). $4 s d e B$ ascospore production was not significantly different to wild type $(p \leqslant 0.05)$ at this temperature.

\subsection{Effect of sdeA and sdeB mutations on A. nidulans radial colony growth at 37 and $22^{\circ} \mathrm{C}$}

The effect of the $\Delta 9$-stearic acid desaturase gene mutations on colony growth was determined by measuring radial colony diameters after five days growth. Fig. 3 shows that at $37^{\circ} \mathrm{C}, \Delta s d e A$ was significantly $(p \leqslant 0.05)$ impaired in growth compared to $\triangle s d e B$ and wild type, which are not significantly different at this temperature. Colony growth of both $\triangle s d e A$ and $\triangle s d e B$ was significantly reduced $(p \leqslant 0.05)$ compared to wild type at $22^{\circ} \mathrm{C}$, with $\Delta s d e A$ proving again to be more severely impaired in growth compared to $\triangle s d e B$ (Fig. 3). The significant $(p \leqslant 0.05)$ reduction in growth of $\triangle s d e B$ at $22{ }^{\circ} \mathrm{C}$, coupled with reduced conidiation compared to wild type at low temperatures, suggests a role for the SdeB protein in the physiological adaptation to cold.

The $\Delta s d e A ; \triangle s d e B$ double mutant was constructed by sexual crossing of the parental disruptant strains. This combination of mutated genes is synthetically lethal and the strain, carrying no intact $\Delta 9$-stearic acid desaturase loci, is incapable of growth or development on unsupplemented GMM or YGT at any temperature (data not shown). Fig. 4 illustrates the qualitative differences and similarities in radial growth of these mutants and wild type at $37^{\circ} \mathrm{C}$.

\subsection{Fatty acid analysis}

Mycelial fatty acid content of the desaturase mutants was assessed using GC analysis. Table 2 shows the total amount of fatty acids produced by each mutant and wild type as well as the proportion of each fatty acid detected in the FAME mixture. In $\Delta s d e A$, total fatty acid content was increased 3.5-fold over the amount produced by wild type. For $\Delta s d e B$, total fatty acid content was reduced to $72 \%$ of the amount produced by wild type. 


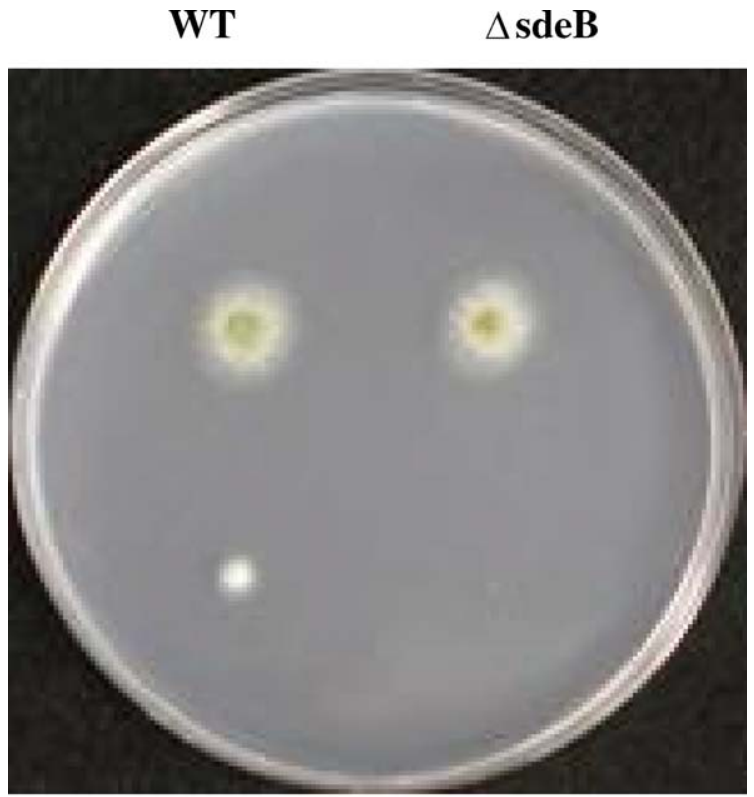

\section{$\Delta$ sdeA}

Fig. 4. Phenotype of desaturase mutants. The effects on growth of the mutations $\Delta s d e A, \Delta s d e B$, wild type, and the $\Delta s d e A ; \Delta s d e B$ double mutant are shown here after growth on GMM for $48 \mathrm{~h}$ at $37^{\circ} \mathrm{C}$.

One action of $\Delta 9$-stearic acid desaturase is to convert palmitic acid to palmitoleic acid. Consequently, accumulation of palmitic acid increased by 125 and $150 \%$ in $\triangle s d e A$ and $\triangle s d e B$, respectively, compared to wild type, while palmitoleic acid content was reduced in $\Delta s d e A$ and $\triangle s d e B$ to 1 and $35 \%$, respectively, of the levels produced by wild type.

The second function of $\Delta 9$-stearic acid desaturases is to convert stearic acid to oleic acid. Disruption of $s d e A$ led to more than a 3-fold increase in stearic acid compared to wild type, while disruption of the $s d e B$ gene increased stearic acid production 1.3-fold. Compared to wild type, oleic acid levels were reduced approximately 4-fold in both disruption strains compared to wild type. Linoleic acid was almost undetectable in $\triangle s d e A$, and reduced 3 -fold in $\triangle s d e B$.

For $\Delta s d e A$, fatty acid desaturation, measured as a ratio of unsaturated to saturated fatty acids, was reduced to only $4.5 \%$ the amount of desaturation seen for

wild type. Total desaturation was reduced to $18 \%$ of the wild type level in $\Delta s d e B$.

\subsection{Feedback regulation of fatty acid metabolism}

RNA transcript accumulation was studied in the $\triangle s d e A, \Delta s d e B$ and wild type strains (Figs. 5A and B). In wild type, at $37^{\circ} \mathrm{C}$, sde $A$ was transcribed at high levels from 36 to $48 \mathrm{~h}$ before diminishing at $72 \mathrm{~h}$. $s d e B$ was expressed at much lower levels than $\operatorname{sde} A$ in wild type, and expression was strongest at $36 \mathrm{~h}$ before diminishing to undetectable levels by $72 \mathrm{~h}$. This suggests different roles for the two $\Delta 9$-stearic acid desaturases under these physiological conditions. At $22^{\circ} \mathrm{C}$ sde $A$ and $s d e B$ expression in wild type was similar to that seen at $37^{\circ} \mathrm{C}$ with the exception that $s d e B$, while still expressed at very low levels, was expressed for longer time points in the wild type (Fig. 5B). Levels of sde $A$ transcript were similar in $\triangle s d e B$ and wild type at both temperatures. However, in $\Delta s d e A$, up-regulation of $s d e B$ expression occurred at both temperatures compared to wild type, with $s d e B$ expression being particularly strong in the $\Delta s d e A$ background at $22{ }^{\circ} \mathrm{C}$. This indicates a degree of rescuing of the $\Delta s d e A$ genetic lesion by $s d e B$. The expression of fas $A$ (Fig. 5), encoding the fatty acid synthase $\alpha$-subunit gene (Brown et al., 1996) was shown to be elevated in $\Delta s d e A$ compared to $\triangle s d e B$ and wild type at both temperatures studied. fas $A$ expression was not altered in $\triangle s d e B$ compared to wild type. Increased expression of fas $A$ in $\triangle s d e A$ could reflect the increase in total fatty acid content seen for $\triangle s d e A$ at $37^{\circ} \mathrm{C}$ in Table 2.

Previous studies have shown that like $\triangle s d e A$, the $\triangle o d e A$ mutation also leads to an increase in total fatty acid production (Calvo et al., 2001). Therefore, the effect of the $\Delta o d e A$ mutation on fas $A$ gene expression was also investigated. fas $A$ was elevated in $\Delta$ ode $A$ to levels intermediate between sde $A$ and wild type as shown in Fig. 6. Because mutations which increase endogenous stearic acid and oleic acid content appear to stimulate fas $A$ gene expression and fatty acid biosynthesis (Table 2, Fig. 6 and Calvo et al., 2001), while linoleic acid is known to down regulate fatty acid biosynthesis (Clarke and Jump, 1993), the individual effects of these lipids on wild type fas $A$ expression was investigated. Stearic acid

Table 2

Fatty acid composition of mycelia of $A$. nidulans wild type (WT), $\Delta s d e A$, and $\Delta s d e B$

\begin{tabular}{|c|c|c|c|c|c|c|c|c|}
\hline \multirow[t]{2}{*}{ Sample } & \multirow[t]{2}{*}{ Total FAMEa } & \multicolumn{6}{|c|}{ Weight $\%$ of individual FAME } & \multirow[t]{2}{*}{ Desaturation $^{\mathrm{b}}$} \\
\hline & & $16: 0$ & $16: 1$ & $18: 0$ & $18: 1$ & $18: 2$ & $18: 3$ & \\
\hline WT & $0.85 \pm 0.32$ & $48.25 \pm 0.85$ & $0.65 \pm 0.15$ & $11.9 \pm 1.2$ & $14.5 \pm 3.3$ & $24.65 \pm 3.75$ & Trace & 0.66 \\
\hline$\Delta s d e A$ & $3.02 \pm 0.5$ & $60.55 \pm 1.45$ & $0.01 \pm 0.01$ & $36.15 \pm 0.05$ & $3.0 \pm 1.5$ & $0.05 \pm 0.05$ & Trace & 0.03 \\
\hline$\Delta s d e B$ & $0.62 \pm 0.04$ & $72.43 \pm 4.51$ & $0.23 \pm 0.15$ & $16.33 \pm 0.9$ & $3.6 \pm 1.62$ & $7.23 \pm 3.62$ & Trace & 0.12 \\
\hline
\end{tabular}

Analysis was carried out on $72 \mathrm{~h}$ old mycelia grown in stationary liquid glucose minimal medium under continuous dark conditions at $37^{\circ} \mathrm{C}$.

a Abbreviations: FAME, fatty acid methyl esters, given as ng FAME per $\mu$ g lyophilized weight of mycelia; 16:0, palmitic acid; 16:1, palmitoleic acid; 18:0, stearic acid; 18:1, oleic acid; 18:2, linoleic acid; and 18:3, linolenic acid. Values are means of four replicates. Standard deviation is shown.

${ }^{b}$ Desaturation represents the ratio of unsaturated to saturated lipids (i.e., 16:1 + 18:1 + 18:2/16:0 + 18:0). 
B

A

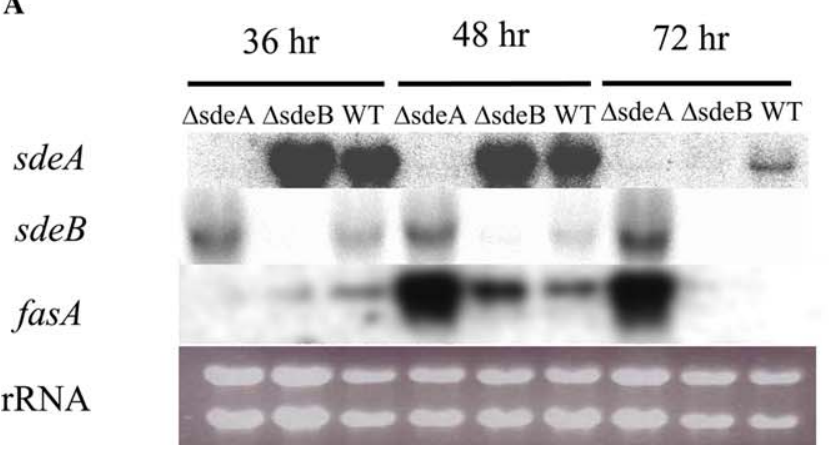

$72 \mathrm{hr}$

$96 \mathrm{hr}$

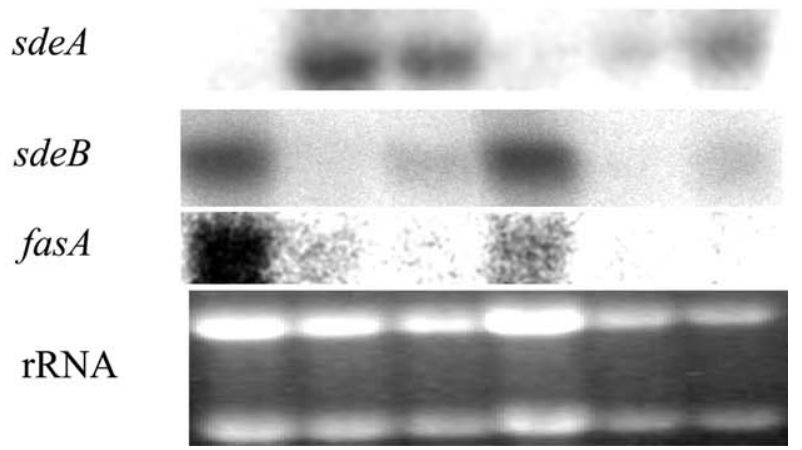

Fig. 5. RNA analysis of $s d e A$, $s d e B$, and $f a s A$ gene expression in $\Delta s d e A, \Delta s d e B$, and wild type (WT) strains after growth at (A) $37^{\circ} \mathrm{C}$ and (B) $22^{\circ} \mathrm{C}$.

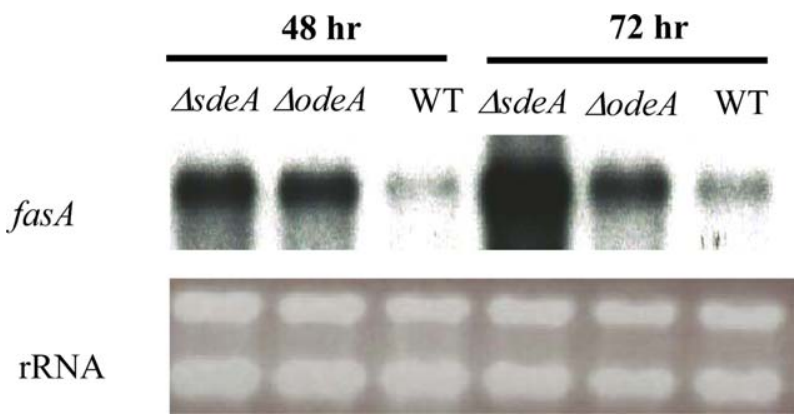

Fig. 6. RNA analysis of fas $A$ gene expression in $\triangle s d e A, \Delta o d e A$, and wild type (WT) strains.

and oleic acid induced fas $A$ gene expression, while linoleic acid had no discernable affect (data not shown). The fluxes in transcript level in both Figs. 5 and 6 support a case for fatty acid feedback regulation of fatty acid metabolism genes, similar to previous reports (Bassilian et al., 2001; Calvo et al., 2001; Clarke and Jump, 1993; Lee et al., 1998).

\subsection{Failure to remediate 49-stearic acid desaturase mutants to wild type growth by addition of exogenous fatty acid}

To determine if wild type phenotype could be restored to the desaturase mutants by exogenous fatty acids, $\Delta s d e A, \Delta s d e B$, wild type, and the $\Delta s d e A ; \Delta s d e B$ double mutant were grown on GMM supplemented with $1 \%(\mathrm{v} / \mathrm{v})$ Tween 80 at 37 and $22^{\circ} \mathrm{C}$ (Fig. 7). Despite supplementation, $\triangle s d e A$ grew significantly less than wild type and $\Delta s d e B(p \leqslant 0.05)$ at 22 and $37^{\circ} \mathrm{C}$, while $\Delta s d e B$ still grew significantly less than wild type $(p \leqslant 0.05)$ at $22^{\circ} \mathrm{C}$. Although none of the mutants were remediated to wild type growth by exogenous unsaturated fatty acids, this is the only condition that allows some growth of the $\Delta s d e A ; \triangle s d e B$ double mutant. Fatty acid analysis of

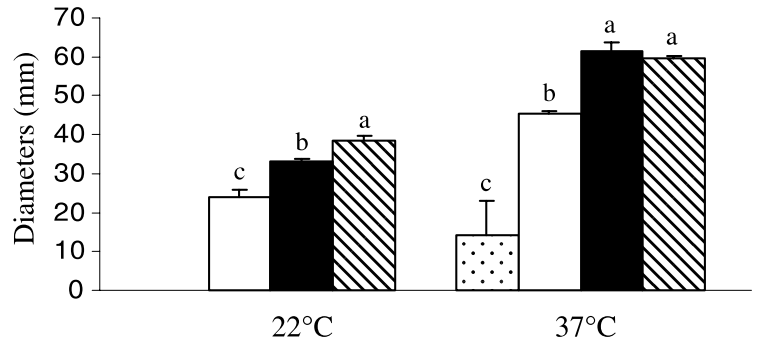

Fig. 7. Remediation of growth. The effect of exogenous oleic acid on strain growth was determined by measuring radial colony diameters of $\triangle s d e A$ (open column), $\Delta s d e B$ (closed column), wild type (hatched column), and $\triangle s d e A ; \triangle s d e B$ (spotted column) grown in the light on GMM supplemented with $1 \%$ (v/v) Tween 80 for $120 \mathrm{~h}$ at 22 and $37^{\circ} \mathrm{C}$. Values for radial colony diameters are the means of three replicates. Columns with the same letter are not significantly different $(p \leqslant 0.05)$. Radial colony diameters at 22 and $37^{\circ} \mathrm{C}$ were not compared.

$\triangle s d e A$ and wild type grown in liquid GMM supplemented with $1 \%(\mathrm{v} / \mathrm{v})$ Tween 80 showed that while the unsaturated fatty acid profile of $\Delta s d e A$ approached that of wild type, stearic acid content is still increased in $\Delta s d e A$ (Table 3).

\section{Discussion}

In a number of fungi, including $N$. crassa (Scott, 1977), Aspergillus niger (Chattopadhyay et al., 1985), and Saccharomyces cerevisiae (Stukey et al., 1989), loss of $\Delta 9$-stearic acid desaturase function is lethal and these mutants require the addition of unsaturated fatty acids for growth. Searches of the relevant genomic databases reveal only one $\Delta 9$-stearic acid desaturase for each of these species (data not shown). A. nidulans more closely resembles Mortierella alpina (MacKenzie et al., 2002) and higher organisms such as mice (Miyazaki et al., 2001 and references therein) which carry multiple $\Delta 9$ stearic acid desaturase genes. Consequently, deletion of 
Table 3

Fatty acid composition of mycelia of $A$. nidulans wild type (WT) and $\Delta s d e A$, and the composition of GMM $+1 \%$ Tween 80 media ${ }^{\text {a }}$

\begin{tabular}{|c|c|c|c|c|c|c|c|}
\hline \multirow[t]{2}{*}{ Sample } & \multicolumn{6}{|c|}{ Weight $\%$ of individual FAME } & \multirow[t]{2}{*}{ Desaturation $^{\mathrm{b}}$} \\
\hline & $16: 0$ & $16: 1$ & $18: 0$ & $18: 1$ & $18: 2$ & $18: 3$ & \\
\hline WT & $10.3 \pm 1.22$ & $2.42 \pm 0.5$ & $3.58 \pm 1.9$ & $49.5 \pm 6.5$ & $29.8 \pm 6.2$ & $4.4 \pm 0.7$ & 6.2 \\
\hline$\Delta s d e A$ & $14.2 \pm 0.9$ & $8.4 \pm 1.9$ & $14.5 \pm 1.3$ & $33.6 \pm 2.6$ & $25.7 \pm 6.1$ & $3.5 \pm 0.7$ & 2.5 \\
\hline Media & 6.49 & 4.37 & 2.0 & 62.7 & 0 & 24.42 & \\
\hline
\end{tabular}

Analysis was carried out on $72 \mathrm{~h}$ old mycelia grown in stationary liquid glucose minimal medium plus $1 \%$ (v/v) Tween 80 under conditions of continuous dark at $37^{\circ} \mathrm{C}$.

a Abbreviations: FAME, fatty acid methyl esters, given as ng FAME per $\mu$ g lyophilized weight of mycelia; 16:0, palmitic acid; 16:1, palmitoleic acid; 18:0, stearic acid; 18:1, oleic acid; 18:2, linoleic acid; and 18:3, linolenic acid. Values are means of four replicates. Standard deviation is shown.

${ }^{\mathrm{b}}$ Desaturation represents the ratio of unsaturated to saturated lipids (i.e., 16:1+18:1+18:2+18:3/16:0 + 18:0).

either $A$. nidulans $\Delta 9$-stearic acid desaturase is tolerated by the organism, while disruption of both $s d e A$ and $s d e B$ genes in a single strain is lethal. However, the two single mutants, $\Delta s d e A$ and $\Delta s d e B$, are not equivalent in their affects on Aspergillus growth and development. The former mutation was the most debilitating, leading to severe reductions in growth, conidiation and ascospore production at 37 and $22^{\circ} \mathrm{C}$. In contrast, $\Delta s d e B$ was indistinguishable from wild type in growth and conidiation at $37{ }^{\circ} \mathrm{C}$, but grew worse and produced less conidia than wild type at $22^{\circ} \mathrm{C}$. Ascospore production was not affected by $\triangle s d e B$ at $22^{\circ} \mathrm{C}$, and $\triangle s d e B$ actually increased ascospore production at $37^{\circ} \mathrm{C}$. Consequently, sde $A$ might play a global role in fitness, while $s d e B$ is required for growth and conidiation at lower temperatures and is important for normal ascospore development at $37^{\circ} \mathrm{C}$. Differences in the role of $s d e A$ and $s d e B$ at physiological temperatures were reflected in Northern analyses. At $37^{\circ} \mathrm{C}$, sde $A$ was expressed strongly in wild type, while $s d e B$ was expressed at much lower levels (Fig. 5A). At $22^{\circ} \mathrm{C}$, sdeB was expressed at lower levels in wild type than $s d e A$, but its expression was detected at later time points compared to $37^{\circ} \mathrm{C}$.

The changes in growth and spore production of $\triangle s d e A$ and $\triangle s d e B$ strains could result from a number of reasons including unusual fatty acid composition of the plasma membrane and/or altered fatty acid ratios. Fatty acid composition could adversely affect membrane fluidity, a factor thought to modulate the dynamics and function of membrane proteins (Baenziger et al., 1999; Tilman and Cascio, 2003) and lead to reduced fungal development. Alternatively, ratios of unsaturated:saturated fatty acids are known to affect Aspergillus and Mucor development (Calvo et al., 1999; Khunyoshyeng et al., 2002), while unsaturated fatty acid derivatives can also influence Aspergillus development (Burow et al., 1997; Calvo et al., 1999; Champe et al., 1987, 1994; Mazur et al., 1990, 1991). Ratios of unsaturated:saturated fatty acids were indeed altered in the $\Delta s d e A$ and $\triangle s d e B$ mutants compared to wild type (see desaturation column, Table 2). Alterations in fatty acid desaturation ratios could then alter downstream derivitization of these unsaturated fatty acids.
The fatty acid profile of $\Delta s d e A$ shows that although stearic- and palmitic acid levels were increased and oleic acid levels were reduced, it still produced measurable amounts of oleic acid, indicating SdeB function. This was supported by elevation of $s d e B$ gene expression in the $\triangle s d e A$ strain at both temperatures studied. The reduction in $\triangle s d e A$ development and growth might be attributed to the delay in $s d e B$ up-regulation seen in $\triangle s d e A$ (Fig. 5A). $\Delta s d e A$ accumulated large amounts of stearic acid, while $\triangle s d e B$ saw a greater increase in palmitic acid production (Table 2). Therefore, one could speculate that under normal growth conditions, sde $A$ encodes a protein whose affinity for stearic acid is higher than palmitic acid, while the SdeB protein is mainly involved with converting palmitic acid to palmitoleic acid.

An additional affect of stearic acid accumulation appears to be stimulation of fatty acid biosynthesis, as seen by the increase in total fatty acids (Table 2) and fas $A$ expression (Fig. 5) for $4 s d e A$ compared to wild type. The increase in total fatty acid content, as seen previously with $\triangle o d e A$, could be attributed to two antagonistic mechanisms. One mechanism would be lack of a negative fatty acid feedback mechanism involving linoleic acid. In $\Delta o d e A$, fas $A$ expression is increased compared to wild type, though not to the same high level seen for $\Delta s d e A$ (Fig. 6). As $\Delta o d e A$ and $\Delta s d e A$ generated only trace amounts of linoleic acid (Calvo et al., 2001; Table 2), this suggests fas $A$ expression is regulated negatively by linoleic acid, as for other organisms (Clarke and Jump, 1993). The second mechanism is through positive feedback involving stearic acid and oleic acid. $\Delta s d e A$ accumulated stearic acid to levels 3 -fold greater than seen for wild type (Table 2), while $\triangle o d e A$ accumulates oleic acid 4-fold compared to wild type (Calvo et al., 2001). In wild type, exogenous stearic acid and oleic acid stimulated fas $A$ expression, while linoleic acid did not increase fas $A$ expression (data not shown). Therefore, fatty acid biosynthesis is likely controlled through negative regulation of fas $A$ expression by linoleic acid, as described for other organisms (Clarke and Jump, 1993; Kim et al., 2002; Xu et al., 1999) and positive regulation by stearic acid and oleic 
acid. This latter observation contrasts with the situation in mammals where exogenous fatty acids, including stearic acid, inhibit de novo lipognesis and the activities of fatty acid synthases (Bassilian et al., 2001; Lee et al., 1998).

The reduced growth capacity of the sde mutants could not be restored to wild type levels by the addition of exogenous oleic acid, as $1 \%(\mathrm{v} / \mathrm{v})$ Tween 80 , even though this addition markedly improved growth of the double mutant (Fig. 7). For $\Delta s d e A$, although levels of oleic acid, linoleic acid, and linolenic acid were approaching wild type when grown on $1 \%(\mathrm{v} / \mathrm{v})$ Tween 80 supplemented media (Table 3 ), indicating cellular uptake and incorporation of exogenous fatty acids, stearic acid content was still elevated and percent desaturation still reduced compared to wild type. Therefore, exogenous fatty acids are unable to prevent stearic acid accumulation, which in turn affects Aspergillus growth.

Taken together, these results suggest a strong genetic link between fatty acid biosynthesis and morphological development in A. nidulans. Continuing investigations of this connection will also reveal interesting insights into the regulation and control of fatty acid metabolism in filamentous fungi.

\section{Acknowledgments}

We thank Dr. Brian Fox, Dr. Robert Zarnowski, and Mr. Enrique Gomez for assistance with fatty acid analysis. This work was funded by NRI 2001-3531910996 and ICRISAT AGR DTD 02/21/01 and in part supported by a specific cooperative agreement, 58-6435081, with USDA-ARS.

\section{References}

Bassilian, S., Ahmed, S., Lim, K.S., Boros, L.G., Mao, C.S., Lee, P.W.-N., 2001. Loss of regulation of lipogenesis in the Zucker diabetic rat. II. Changes in stearate and oleate synthesis. Am. J. Physiol. Endocrinol. Metab. 282, E507-E513.

Baenziger, J.E., Darsaut, T.E., Morris, M.L., 1999. Internal dynamics of the nicotinic acetylcholine receptor in reconstituted membranes. Biochemistry 38, 4905-4911.

Bligh, E.G., Dyer, W.J., 1959. A rapid method of total lipid extraction and purification. Can. J. Biol. Chem. Physiol. 37, 911-917.

Brown, D.W., Adams, T.H., Keller, N.P., 1996. Aspergillus has distinct fatty acid synthases for primary and secondary metabolism. Proc. Natl. Acad. Sci. USA 93, 14873-14877.

Burow, G.B., Nesbitt, T.C., Dunlap, J., Keller, N.P., 1997. Seed lipoxygenase products modulate Aspergillus mycotoxin biosynthesis. Mol. Plant Microbe Interact. 10, 380-387.

Calvo, A.M., Gardner, H.W., Keller, N.P., 2001. Genetic connection between fatty acid metabolism and sporulation in Aspergillus nidulans. J. Biol. Chem. 276, 25766-25774.

Calvo, A.M., Hinze, L.L., Gardner, H.W., Keller, N.P., 1999. Sporogenic effect of polyunsaturated fatty acids on development of Aspergillus spp. Appl. Environ. Microbiol. 65, 3668-3673.
Champe, S.P., Nagle, D.L., Yager, L.N., 1994. Sexual sporulation. Prog. Ind. Microbiol. 29, 429-454.

Champe, S.P., Rao, P., Chang, A., 1987. An endogenous inducer of sexual development in Aspergillus nidulans. J. Gen. Microbiol. 133, $1383-1388$

Chattopadhyay, P., Banerjee, S.K., Sen, K., Chakrabarti, P., 1985. An unsaturated fatty acid mutant of Aspergillus niger with partially defective delta 9-desaturase. Can. J. Microbiol. 31, 346-351.

Clarke, S.D., Jump, D.B., 1993. Regulation of gene transcription by polyunsaturated fatty acids. Prog. Lipid Res. 32, 139-149.

Evans, J.L., Moclock, M.A., Gealt, M.A., 1986. The fatty acid composition of the conidia and mycelia of the fungus Aspergillus nidulans. Can. J. Microbiol. 32, 179-181.

Khunyoshyeng, S., Cheevadhanarak, S., Rachdawong, S., Tanticharoen, M., 2002. Differential expression of desaturases and changes in fatty acid composition during sporangiospore germination and development in Mucor rouxii. Fungal Genet. Biol. 37, 13-21.

Kim, H.-J., Miyazaki, M., Ntambi, J.M., 2002. Dietary cholestorol opposes PUFA-mediated repression of the stearoyl-CoA desaturase-1 gene by SREBP-1 independent mechanism. J. Lipid Res. 43, 1750-1757.

Lee, P.W.-N., Lim, S., Bassilian, S., Bergner, E.A., Edmond, J., 1998. Fatty acid cycling in human hepatoma cells and the effects of troglitazone. J. Biol. Chem. 272, 20929-20934.

Los, D.A., Murata, N., 1998. Structure and expression of fatty acid desaturases. Biochim. Biophys. Acta 1394, 3-5.

MacKenzie, D.A., Carter, A.T., Wongwathanarat, P., Eagles, J., Salt, J., Archer, D.B., 2002. A third fatty acid Delta9-desaturase from Mortierella alpina with a different substrate specificity to ole $1 \mathrm{p}$ and ole2p. Microbiology 148, 1725-1735.

Mazur, P., Meyers, H.V., Nakanishi, K., 1990. Structural elucidation of sporogenic fatty acid metabolites from Aspergillus nidulans. Tetrahedron Lett. 31, 3837-3840.

Mazur, P., Nakanishi, K., El-Zayat, A.A.E., Champe, S.P., 1991. Structure and synthesis of sporogenic psi factors from Aspergillus nidulans. J. Chem. Soc. Chem. Commun. 20, 1486-1487.

Miyazaki, M., Man, W.C., Ntambi, J.M., 2001. Targeted disruption of stearoyl-CoA desaturasel gene in mice causes atrophy of sebaceous and meibomian glands and depletion of wax esters in the eyelid. J. Nutr. 131, 2260-2268.

Oakley, B.R., Rinehart, J.E., Mitchell, B.L., Oakley, C.E., Carmona, C., Gray, G.L., May, G.S., 1987. Cloning, mapping and molecular analysis of the $\operatorname{pyr} G$ (orotidine- $5^{\prime}$-phosphate decarboxylase) gene of Aspergillus nidulans. Gene 61, 385-399.

Pontecorvo, G., Roper, J.A., Hemmons, L.M., MacDonald, K.D., Bufton, A.W., 1953. The genetics of Aspergillus nidulans. Adv. Genet. 5, 141-263.

Sambrook, J., Fritsch, E.F., Maniatis, T., 1989. Molecular Cloning: A Laboratory Manual, second ed. Cold Spring Harbor laboratory Press, Plainview, NJ.

Scott, W.A., 1977. Mutations resulting in an unsaturated fatty acid requirement in Neurospora. Evidence for delta9-desaturase defects. Biochemistry 16, 5274-5280.

Sienko, M., Paszewski, A., 1999. The met $G$ gene of Aspergillus nidulans encoding cystathionine beta-lyase: cloning and analysis. Curr. Genet. 35, 628-646.

Stukey, J.E., McDonough, V.M., Martin, C.E., 1989. Isolation and characterization of OLE1, a gene affecting fatty acid desaturation from Saccharomyces cerevisiae. J. Biol. Chem. 264, 16537-16544.

Tilman, T.S., Cascio, M., 2003. Effects of membrane lipids on ion channel structure and function. Cell Biochem. Biophys. 38, 161190

Xu, J., Nakamura, M.T., Cho, H.P., Clarke, S.D., 1999. Sterol regulatory element binding protein-1 expression is suppressed by dietary polyunsaturated fatty acids. A mechanism for the coordinate suppression of lipogenic genes by polyunsaturated fats. J. Biol. Chem. 274, 23577-23583. 